\title{
Phylogenetic Tree Analysis of the Cold-Hot Nature of Traditional Chinese Marine Medicine for Possible Anticancer Activity
}

\author{
Xianjun Fu, ${ }^{1}$ Xuxia Song, ${ }^{2}$ Xuebo Li, ${ }^{1}$ Kah Keng Wong, ${ }^{3}$ Jiaoyang Li, ${ }^{1}$ Fengcong Zhang, \\ Changyun Wang, and Zhenguo Wang ${ }^{1}$
}

\author{
${ }^{1}$ School of Information Management, Shandong University of Traditional Chinese Medicine, Jinan 250355, China \\ ${ }^{2}$ Department of Neurology, Qingdao Municipal Hospital, Qingdao 266011, China \\ ${ }^{3}$ Department of Immunology, School of Medical Sciences, Universiti Sains Malaysia, Health Campus, \\ 16150 Kubang Kerian, Kelantan, Malaysia \\ ${ }^{4}$ Key Laboratory of Marine Drugs, Ministry of Education of China, School of Medicine and Pharmacy, \\ Ocean University of China, Qingdao, Shandong Province 266003, China
}

Correspondence should be addressed to Xianjun Fu; xianxiu@hotmail.com and Zhenguo Wang; zhenguow@126.com

Received 30 August 2016; Revised 30 October 2016; Accepted 4 December 2016; Published 12 January 2017

Academic Editor: Thierry Hennebelle

Copyright (C) 2017 Xianjun Fu et al. This is an open access article distributed under the Creative Commons Attribution License, which permits unrestricted use, distribution, and reproduction in any medium, provided the original work is properly cited.

Traditional Chinese Marine Medicine (TCMM) represents one of the medicinal resources for research and development of novel anticancer drugs. In this study, to investigate the presence of anticancer activity (AA) displayed by cold or hot nature of TCMM, we analyzed the association relationship and the distribution regularity of TCMMs with different nature (613 TCMMs originated from 1,091 species of marine organisms) via association rules mining and phylogenetic tree analysis. The screened association rules were collected from three taxonomy groups: (1) Bacteria superkingdom, Phaeophyceae class, Fucales order, Sargassaceae family, and Sargassum genus; (2) Viridiplantae kingdom, Streptophyta phylum, Malpighiales class, and Rhizophoraceae family; (3) Holothuroidea class, Aspidochirotida order, and Holothuria genus. Our analyses showed that TCMMs with closer taxonomic relationship were more likely to possess anticancer bioactivity. We found that the cluster pattern of marine organisms with reported AA tended to cluster with cold nature TCMMs. Moreover, TCMMs with salty-cold nature demonstrated properties for softening hard mass and removing stasis to treat cancers, and species within Metazoa or Viridiplantae kingdom of cold nature were more likely to contain AA properties. We propose that TCMMs from these marine groups may enable focused bioprospecting for discovery of novel anticancer drugs derived from marine bioresources.

\section{Introduction}

The nature of traditional Chinese medicines (TCMs) can be classified into three categories (cold, hot, and neutral) that represent the types of body reactions after the administration of specific TCM $[1,2]$. The therapeutic effect of CMs depends mainly on the nature of the drugs as well as the processes they regulate to recover the balance between Yin and Yang in the human body $[3,4]$. According to TCM, the rationale for the correct remedy selection is based upon a corresponding TCM syndrome (Zheng, 证, or pattern) [5]. A patient will present with a syndrome upon disruption of Yin-Yang balance, which may be caused either by external and/or internal pathological factors. This can be regarded as clinical phenotype, such as cold or hot syndrome $[5,6]$. The standard therapeutic guideline used to treat cold or hot syndrome is to "cure cold syndrome by medication with hot nature" and to "cure hot syndrome by medication with cold nature" [5]. This therapeutic practice has been validated and developed over thousands of years, and most CMs have thus been labeled with different nature types as an outcome of this repeated clinical practice [1]. Recent literature shows that different biological effects conferred by a specific TCM could serve as the basis to discriminate cold and hot nature of TCMs 
$[7,8]$. Thus, conversely, the cold-hot nature of a specific TCM could potentially serve as clues to its bioactivities including anticancer properties.

As a vital part of TCMs, Traditional Chinese Marine Medicine (TCMM) has been used to treat and prevent diseases for thousands of years, and it is based on a unique theoretical framework, diagnosis, and treatment [9]. TCMM appears to be effective in treating various diseases such as cancers, malaria, diabetes, cardiocerebrovascular diseases, immunodeficiency diseases, and senile dementia, and therefore has become an important medicinal resource for the research and development of new drugs [10].

Cancer poses serious threat to human health worldwide, and there have been efforts in screening for compounds possessing anticancer activity (AA) from TCMMs [17]. Marine organisms including TCMMs have evolved efficient and highly potent metabolites that exhibit strong biological activity at low concentrations to circumvent rapid dilution caused by their aqueous environment [18], and this confers a potential advantage over metabolites of terrestrial origin including TCMs originating from nonmarine sources. Moreover, TCMMs contain significant differences from TCMs of terrestrial origin including their bioactivity properties, cold and hot nature $[10,19,20]$. Marine organisms have been demonstrated to be promising source of novel antitumor compounds $[18,21]$ and several of the marine families of TCMMs have been explored and reportedly show anticancer potential [10, 22].

High-throughput screening for novel anticancer drugs are widely conducted; however it is costly and might yield chemical hits with low actual clinical efficacy and/or high toxicity [23]. It has been reported that distinct plant species yields potent bioactive compounds at higher rates than other plant species, and most drugs are derived from preexisting drug-productive families [24]. Clues to drug-productive species can be obtained from the species-distribution profiles of phylogenetic tree [19]. Almost $80 \%$ of the approved drugs and $67 \%$ of the clinical-trial drugs concentrated in 17 and 30 drug-prolific families, respectively, including Fabid and Malvid groups of the Rosidae subclass, the Lamiid and Campanulid groups of the Asterid subclass, and the Ranunculales order [24]. Eribulin mesylate is a structurally simplified synthetic analogue of halichondrin B used for the treatment of metastatic breast cancer, which is a natural product isolated from the marine sponge Halichondria okadai, which originates from a drug-productive family Halichondriidae [25-27]. Taken together, these instances provide the basis to screen for natural resources possessing AA activities through phylogenetic tree analysis.

Thus, in this study, in order to examine the phylogenetic tree and cold-hot nature of TCMM for identifying TCMMs with potential AA properties, association rules mining and phylogenetic tree construction methodologies were used to investigate the association relationship and distribution regularity of TCMMs with different nature possessing AA properties.

\section{Materials and Methods}

2.1. Datasets Preparation. The cold-hot nature categorization of 613 TCMMs related to 1,091 marine bioresources species were retrieved from the "Chinese Marine Materia Medica" [22]. Latin name and taxonomy data of the related bioresources were retrieved from the National Center for Biotechnology Information (NCBI) Taxonomy Database (https://www.ncbi.nlm.nih.gov/taxonomy) [28]. The 1,091 marine species were clustered into three groups: cold, hot, and neutral.

The anticancer bioactivity information of each marine bioresources species were retrieved from PubMed literature database by using the following retrieval formula.

"Latin name of each marine bioresource species" [All Fields] AND (anticancer [All Fields] OR antitumer [All Fields] OR antitumor [All Fields]).

Each species was labeled with presence or absence of anticancer bioactivity according to the retrieved results. All the results were independently checked by two researchers, $\mathrm{F}$. X. and S. X.

2.2. Phylogenetic Tree Construction. The phylogenetic trees were generated by using the NCBI taxonomy-based automatic tree generator against known families in the Bacteria, Viridiplantae, and Metazoa kingdoms or superkingdoms [24, 29].

First, TAX ID of each marine bioresources species were retrieved from The NCBI Taxonomy System (https://www .ncbi.nlm.nih.gov/taxonomy) [28]. Then data of TAX ID were input to phylot web (http://phylot.biobyte.de/index.html) to construct phylogenetic tree, and the visualization of the tree was conducted in iTOL (version 3.3.2) and EvolView [19, 24, 30, 31]. Family or species names were labeled at branch ends. Reported anticancer bioactivity clusters and different nature were labeled or marked in the phylogenetic trees.

2.3. Association Rules Mining. The association relationship between reported anticancer bioactivity and taxonomy or nature types was mined by aRules package [32] based on the $\mathrm{R}$ platform to elucidate the association rules.

An association rule is an implication of the form $X \Rightarrow Y$, where $X \subset I, Y \subset I$, and $X \cap Y=\varnothing$. The rule $X \Rightarrow Y$ holds in the database $D$ with confidence and support [33]. The support is a measure of the frequency of a rule, and the confidence is a measure of the strength of the relation between sets of items [34]. In this study, the cold-hot nature and taxonomy data of TCMMs were taken as $X$, while the AA of each TCMM was regarded to $Y$; the association rules whose confidence and support were larger than the set thresholds (50.00\% for confidence and $0.5 \%$ for support) were chosen as strong association rules.

\section{Results and Discussion}

3.1. Cluster Pattern of Marine Organism from TCMMs with Reported $A A$. In this study, 613 TCMMs originated from 1,091 species of marine organisms were screened for potential AA properties (Table 1). Majority of the species $(n=870$ of 1,$091 ; 79.74 \%$ ) were from Metazoa kingdom. Among the 1,091 species investigated, 194 species were reported to have AA with nearly half of them $(n=92 ; 47.42 \%)$ from the 
TABLE 1: The distribution of marine species with AA in different kingdom or superkingdom.

\begin{tabular}{|c|c|c|}
\hline Kingdom or Superkingdom ${ }^{\#}$ & Number of species $(\%)^{\mathrm{a}}$ & Number of species with AA $(\%)^{\mathrm{b}}$ \\
\hline Metazoa & $870(79.74 \%)$ & $92(10.57 \%)$ \\
\hline Viridiplantae & $89(8.16 \%)$ & $45(50.56 \%)$ \\
\hline Others in Eukaryota & $123(11.27 \%)$ & $51(41.46 \%)$ \\
\hline Bacteria $^{\#}$ & $9(0.82 \%)$ & $6(66.67 \%)$ \\
\hline Total & $1,091(100 \%)$ & $194(17.78 \%)$ \\
\hline
\end{tabular}

${ }^{a}$ Percentage derived from division with the total number of species within all kingdom and superkingdom combined $(n=1,091)$.

${ }^{\mathrm{b}}$ Percentage derived from division with the number of species within each corresponding kingdom or superkingdom.

\# refers to superkingdom.

Metazoa kingdom. More than half of the interrogated species within the Viridiplantae (green plants) kingdom ( $n=45$ of $89 ; 50.56 \%)$ and Bacteria superkingdom $(n=6$ of 9 ; $66.67 \%$ ) demonstrated AAs. This implies that the marine species of TCMMs from Viridiplantae kingdom and Bacteria superkingdom are more likely to possess AA.

Metazoa kingdom showed 61 AA families concentrated in 38 clusters (Figure 1; Table S1 in Supplementary Material available online at https://doi.org/10.1155/2017/4365715). These families were present in seven of eight phylums known to possess AAs (Table S2). Four phylums contained more than ten AA species including 24 in Mollusca, 23 in Echinodermata, 22 in Chordata, and 10 in Cnidaria. There were 18 AA classes from 25 known classes of Metazoa (Table S3), and three classes (Holothuroidea, Actinopteri, and Bivalvia) contained more than ten AA species. Three orders (Aspidochirotida, Veneroida, and Alcyonacea) contained more than five AA species (Table S4).

This implies that TCMMs from Metazoa are potential candidates for anticancer drug discovery. Diverse peptides with a wide range of biological activities including antimicrobial and antitumoral have been isolated from different phyla of Mollusca, Cnidaria, and Echinodermata [35]. Two novel marine anticancer compounds, kahalalide F and ES285, have been isolated from the Indopacific mollusc Elysia rufescens and the North Atlantic mollusc Spisula polynyma, respectively [36]. The phylum Cnidaria is unique such that practically all of its members are toxic and contain Cnidarian toxins which are a rich source of polypeptides with a wide variety of biological activities including pore-forming cytolysins, phospholipases, neurotoxins, and protease inhibitors [35]. These marine organisms could be an important source of structurally bioactive secondary metabolites. There have been 12 reported novel and highly potent antitumor natural products derived from seven species of cnidarians of marine origin [37].

Figure 2 presents the distribution of marine families with AA in phylogenetic tree of Viridiplantae kingdom, Eukaryota superkingdom, and Bacteria superkingdom. A total of $52 \mathrm{AA}$ families were concentrated in 18 clusters (Figure 2; Table S5). These families were distributed in five AA phylums (Table S6) in which Streptophyta phylums of Viridiplantae contained 35 AA species. There were two classes from the Eukaryota superkingdom containing more than 20 AA species (Table S7), the Florideophyceae (24 AA species) and Phaeophyceae
(23 AA species). One order (Fucales) contained more than ten AA species (Table S8).

Compared with Figure 1, Figure 2 showed more concentrated anticancer family clusters in Bacteria and Eukaryota superkingdom than Metazoa kingdom. Bacteria have widely contributed to some of the most useful chemotherapeutic drugs [38], while marine cyanobacteria contain antiproliferative properties, yielding several potent inhibitors of malignancies [39]. All of the six AA species of TCMMs from Bacteria superkingdom are of Cyanobacteria phylum.

Viridiplantae (green plants) are an ancient group of eukaryotes comprising of two main clades: the Chlorophyta and the Streptophyta. The former consists of a wide diversity of green algae while the latter consists of freshwater green algae and terrestrial plants [40]. There are four phyla of algae including red algae (Florideophyceae), brown algae (Phaeophyceae), green algae (Chlorophyta), and diatom (Bacillariophyceae) and two phyla of plants from coastal wetlands including Pteridophyta and Angiospermae [10]. Marine plants serve as main sources of potential anticancer agents [38].

3.2. Cold-Hot Nature Distribution of Marine Organism from TCMMs with Reported AA. Within the 1,091 marine organisms, 380 can be grouped into TCMMs with cold nature, 233 with hot nature, and 366 with neutral nature (Table 2). More than half of AA species were from the cold group ( $n=51.03 \%)$, followed by the neutral $(25.26 \%)$ and hot group $(12.37 \%)$.

It was reported that basic pharmacological effects of herbals with cold nature are antibacterial, anti-inflammatory, antitumor, antipyretic, diuretic, lowering blood pressure, sedation, and analgesic [41]. Most frequently used TCMMs are generally of cold nature [20]. Studies have shown that salty flavor and cold nature (such as Sargassum and Laminariae Thallus) are representative of TCMMs [10, 20]. In terms of medicinal effects, the most representative efficacies of TCMMs with salty-cold flavor and nature (e.g., Sargassum, Laminariae Thallus, Ostreae Concha, and Meretricis Concha) include softening hard mass and removing stasis to treat cancers [10]. This might serve as an explanation, at least partially, as to why AA species are often from the cold group.

3.3. The Association Rules and Phylogenetic Tree of Marine Organisms. The association rules mining resulted in 12 


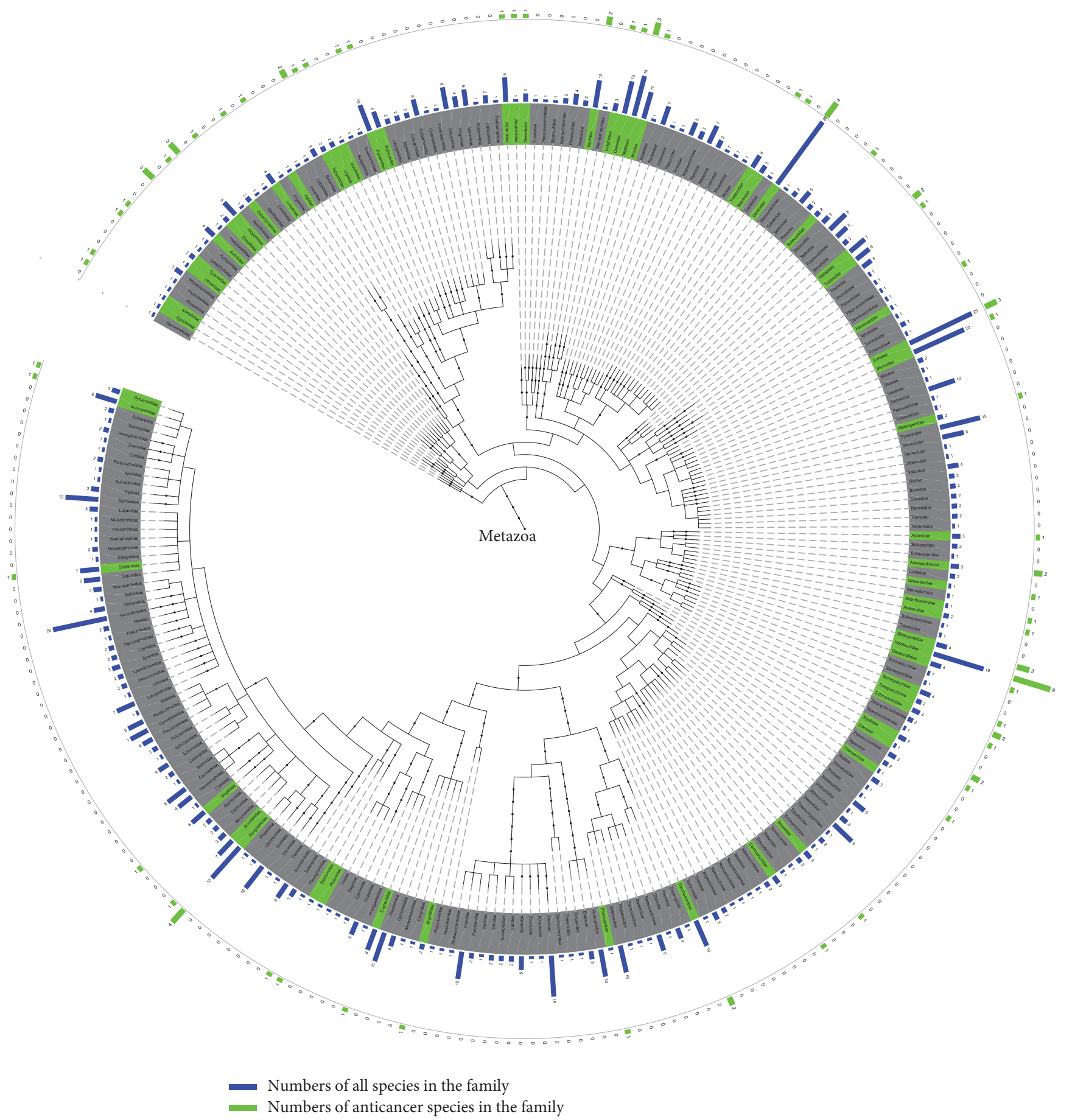

FIGURE 1: The distribution of marine anticancer activity families (green background color) phylogenetic tree of Metazoa kingdom. The family names are provided at branch ends, which can be viewed more clearly by enlarging the figure in the electronic version. The length of the blue and green bar outside the circle represents the number of all species and the AA species, respectively, in the family.

screened rules (Table 3 ). There were 11 rules with single item and one with double items. In the single item rules, one was of superkingdom (Bacteria with confidence of $66.67 \%$ ) and one was associated with Streptophyta phylum, while two were related to Holothuroidea and Phaeophyceae classes. The Malpighiales order and Rhizophoraceae family showed strong association with AA with confidences of
$87.50 \%$ and $85.71 \%$, respectively. The double items of cold and Viridiplantae kingdom also showed strong association with AA, implying that the species of TCMMs with cold nature from Viridiplantae kingdom tend to have AA.

Figure 3 shows the cluster pattern of marine AA families, in the phylogenetic tree of marine organisms, tended to cluster with cold nature TCMMs. In contrast, few of 


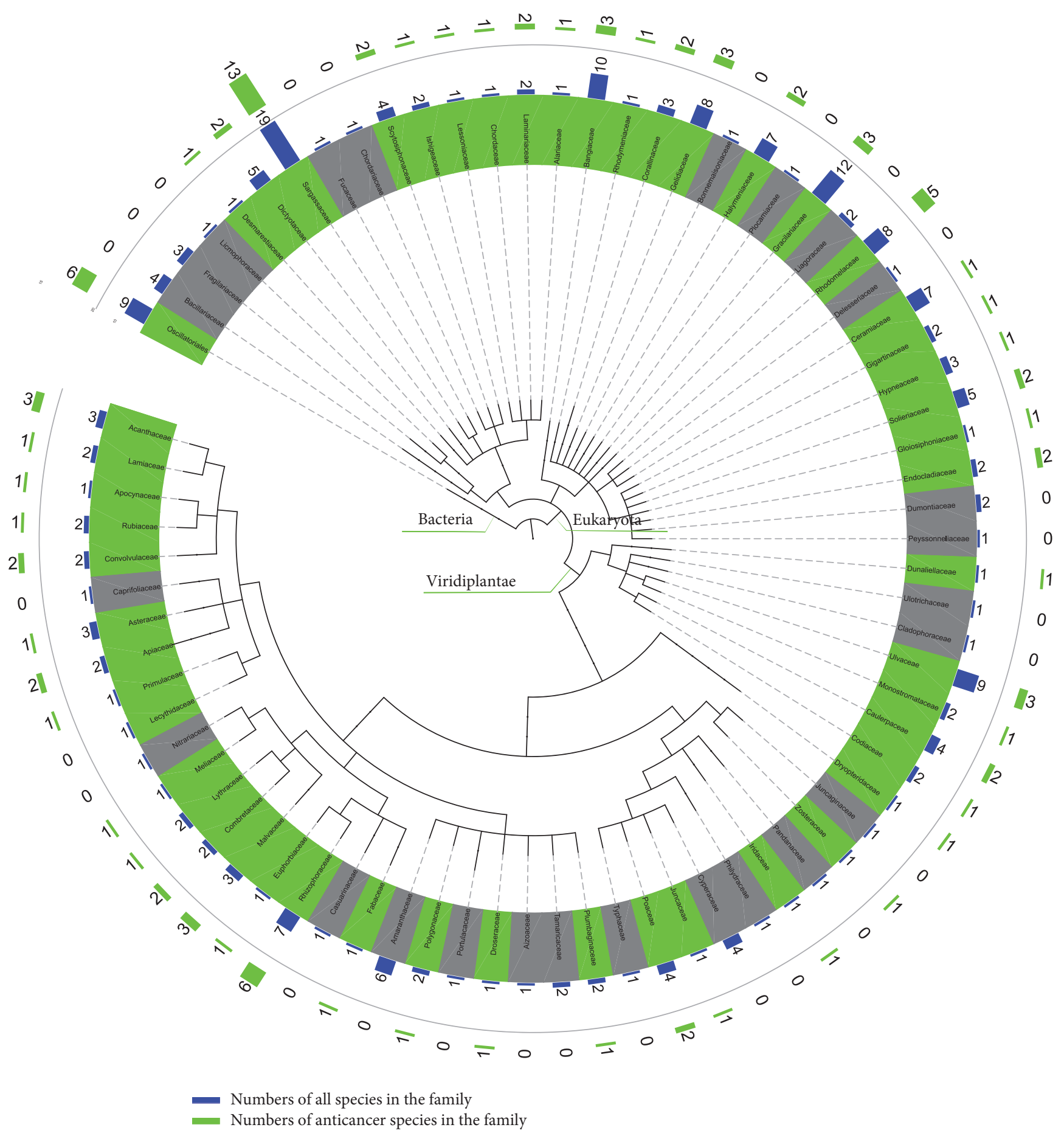

FIGURE 2: The distribution of marine families with AA in phylogenetic tree of Viridiplantae kingdom, Eukaryota superkingdom, and Bacteria superkingdom. Coloring and labeling schemes are as described in Figure 1.

the AAs-containing families clustered with hot and neutral nature TCMMs. The screened association rules labeled at the corresponding branch collected at three species groups (from superkingdom to genus) in the phylogenetic tree. The cluster pattern (Figure 3) contained three major groups: (1) the first group consisting of Bacteria superkingdom, Phaeophyceae class, Fucales order, Sargassaceae family, and Sargassum genus; (2) the second group consisting of Viridiplantae kingdom, Streptophyta phylum, Malpighiales class, and Rhizophoraceae family; (3) the third group comprising three levels of Holothuroidea class, Aspidochirotida order, and Holothuria genus at the same branch.

The results above suggest that certain species of TCMMs with closer taxonomic relationship are more likely to have 
TABLE 2: The distribution of marine organisms with AA in different nature categories.

\begin{tabular}{lcr}
\hline Nature & Number of species $(\%)^{\mathrm{a}}$ & ${\text { Number of species with AA }(\%)^{\mathrm{b}}}^{\mathrm{b}}$ \\
\hline Cold & $380(34.83)$ & $99(26.05)$ \\
Hot & $233(21.36)$ & $24(10.30)$ \\
Neutral & $366(33.55)$ & $49(13.39)$ \\
None & $112(10.27)$ & $22(19.64)$ \\
Total & $1,091(100.00)$ & $194(17.78)$ \\
\hline
\end{tabular}

${ }^{a}$ Percentage derived from division with the total number of species within all different natures combined $(n=1,091)$.

${ }^{\mathrm{b}}$ Percentage derived from division with the number of species within each corresponding nature.

TABLE 3: The results of association rules mining (support $>0.5 \%$, confidence $>50 \%$, lift $>2$ ).

\begin{tabular}{|c|c|c|c|c|c|c|}
\hline Rule ID & Single or double items & Taxonomic rank & Rules & Support & Confidence & Lift \\
\hline 1 & Single item & Super kingdom & $\{$ Bacteria $\} \Rightarrow\{$ anticancer $\}$ & $0.55 \%$ & $66.67 \%$ & 3.75 \\
\hline 2 & Single item & Phylum & $\{$ Streptophyta $\} \Rightarrow$ anticancer $\}$ & $3.21 \%$ & $52.24 \%$ & 2.94 \\
\hline 3 & \multirow{2}{*}{ Single item } & \multirow{2}{*}{ Class } & $\{$ Phaeophyceae $\} \Rightarrow$ anticancer $\}$ & $2.20 \%$ & $63.16 \%$ & 3.55 \\
\hline 4 & & & $\{$ Holothuroidea $\} \Rightarrow\{$ anticancer $\}$ & $1.28 \%$ & $51.85 \%$ & 2.92 \\
\hline 5 & \multirow{3}{*}{ Single item } & \multirow{3}{*}{ Order } & $\{$ Malpighiales $\} \Rightarrow\{$ anticancer $\}$ & $0.64 \%$ & $87.50 \%$ & 4.92 \\
\hline 6 & & & $\{$ Fucales $\} \Rightarrow\{$ anticancer $\}$ & $1.19 \%$ & $65.00 \%$ & 3.66 \\
\hline 7 & & & $\{$ Aspidochirotida $\} \Rightarrow$ anticancer $\}$ & $1.10 \%$ & $52.17 \%$ & 2.93 \\
\hline 8 & \multirow{2}{*}{ Single item } & \multirow{2}{*}{ Family } & $\{$ Rhizophoraceae $\} \Rightarrow$ anticancer $\}$ & $0.55 \%$ & $85.71 \%$ & 4.82 \\
\hline 9 & & & $\{$ Sargassaceae $\} \Rightarrow$ anticancer $\}$ & $1.19 \%$ & $68.42 \%$ & 3.85 \\
\hline 10 & \multirow{2}{*}{ Single item } & \multirow{2}{*}{ Genus } & $\{$ Sargassum $\} \Rightarrow\{$ anticancer $\}$ & $1.01 \%$ & $68.75 \%$ & 3.87 \\
\hline 11 & & & $\{$ Holothuria $\} \Rightarrow\{$ anticancer $\}$ & $0.73 \%$ & $61.54 \%$ & 3.46 \\
\hline 12 & Double items & Nature \& kingdom & $\{$ cold, Viridiplantae $\} \Rightarrow\{$ anticancer $\}$ & $2.57 \%$ & $56.00 \%$ & 3.15 \\
\hline
\end{tabular}

AA. The AA of marine organisms is mostly based on the secondary metabolites of each species $[42,43]$. The distribution of secondary metabolites has some value for taxonomy [44]. Chemical structure of secondary metabolites forms the molecular basis for its bioactivity [45], and marine natural products are important sources of chemical scaffolds [46]. Natural products from marine species with closer taxonomic relationship contain similar scaffolds and bioactivities [47]. For instance, the marine organisms Sargassum fusiforme, Sargassum hemiphyllum, Sargassum pallidum, Sargassum carpophyllum, Sargassum horneri, and Sargassum thunbergii are from the Sargassaceae family that form the TCMM seaweed (known as “haizao" in Chinese or 海藻) with cold nature. All of the Sargassum seaweed possessed phytosterols compounds with the same scaffold (compounds $1-5$ in Table 4) or similar structure (compounds 6 in Table 4) containing similar anticancer bioactivity.

In addition to the grouped species of TCMMs with closer taxonomic relationship, TCMM species with cold nature from Viridiplantae kingdom also showed a tendency for AA with confidence of $56 \%$. As discussed above, marine plants contain potential anticancer agents and cold nature TCMMs render softening of hard mass and stasis removal. Hence, the species (e.g., Ulva pertusa [48]) with combination of Viridiplantae kingdom and cold nature are more likely to demonstrate AA. For example, Ulva pertusa from the Ulvaceae family of Viridiplantae kingdom was used as classic TCMM with cold nature to treat thyroid neoplasm from
Tang dynasty and recorded in the herbal book of "Bencao Shiyi (Supplement to Materia Medica, 本草拾遗)” [22]. It is reported that Ulva pertusa showed antitumor activity against Meth-A fibrosarcoma by intraperitoneal administration of $50 \mathrm{mg} / \mathrm{kg}$ daily for seven days [49].

Nonetheless, we acknowledge limitations of the study as follows: (1) the studies included in the phylogenetic tree analysis took into account results from in vitro investigations. However, in vitro studies have remained the prerequisite before a candidate compound is tested further in in vivo or human trials settings, and excluding results from in vitro studies could significantly reduce the sensitivity of our analysis; (2) this study represented TCMMs originated from species of marine organisms for potential AA properties available currently and several more are being actively discovered.

Our previous study showed that the TCMM from the organisms in the same family may have the same nature, while marine plants such as Chlorophyta, Florideophyceae, and Phaeophyceae were associated with cold nature, and marine animals including Decapoda, Malacostraca, and Arthropoda contained close relationship with hot nature [19]. The different nature types seem to affect different biological processes based on the pluralistic character of molecular structure [50]. For distribution of secondary metabolites from marine species with closer taxonomic relationship, they contain similar scaffolds and bioactivities [47]. Marine algae associated 


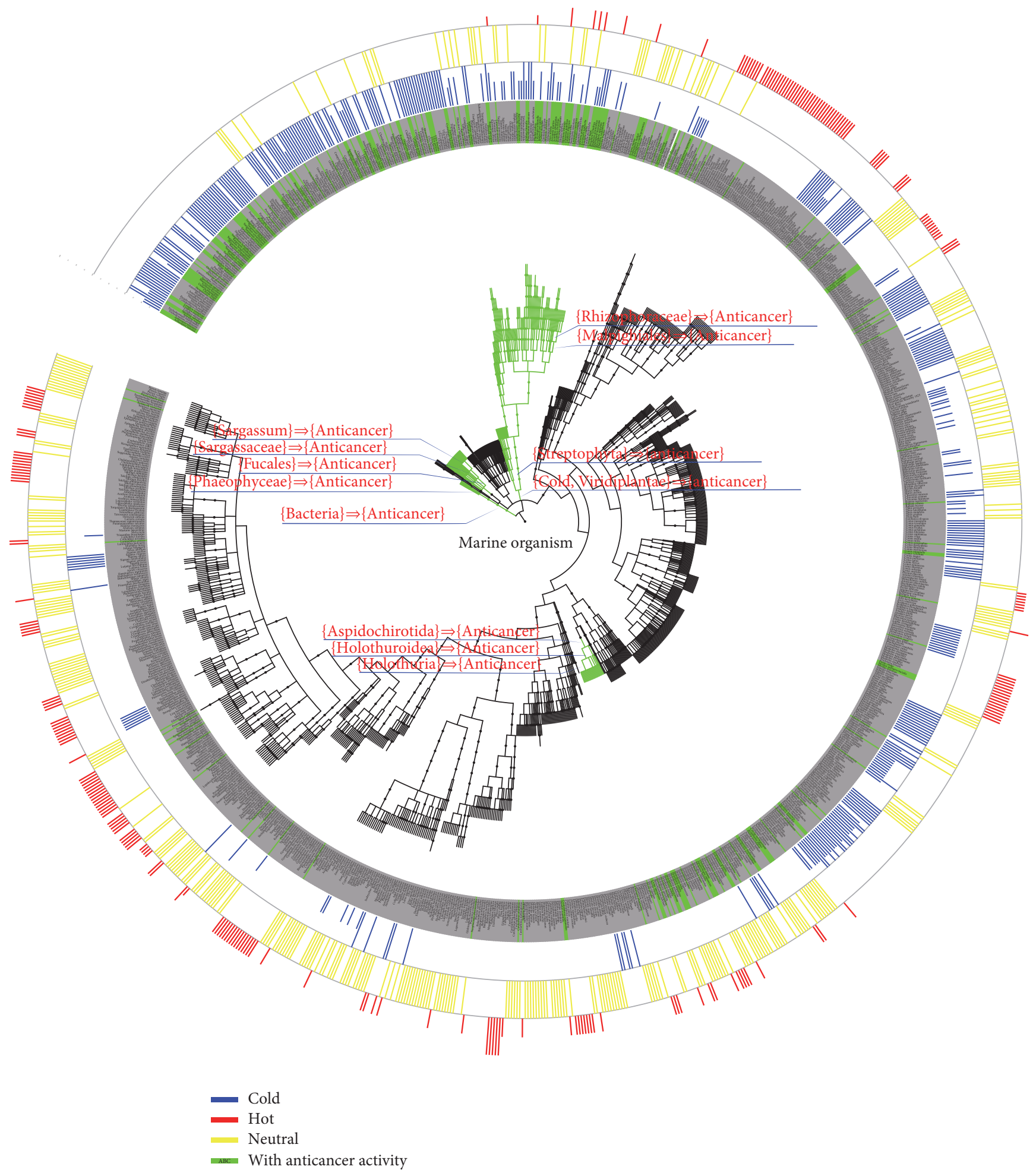

FIGURE 3: The distribution of marine AA species (green background) in the phylogenetic tree of marine organisms. The cold-hot nature of each species was labeled outside of the circle by different colors. The length of the bar represents the degree of nature of TCMMs. The screened association rules were labeled at the corresponding branch (the branches marked with different color). The names of species of the marine organisms of TCMMs are provided at branch ends, which can be viewed more clearly by enlarging the figure in the electronic version. 
TABLE 4: Anticancer compounds from species of Sargassaceae family of the TCMM Seaweed (Haizao, 海藻).

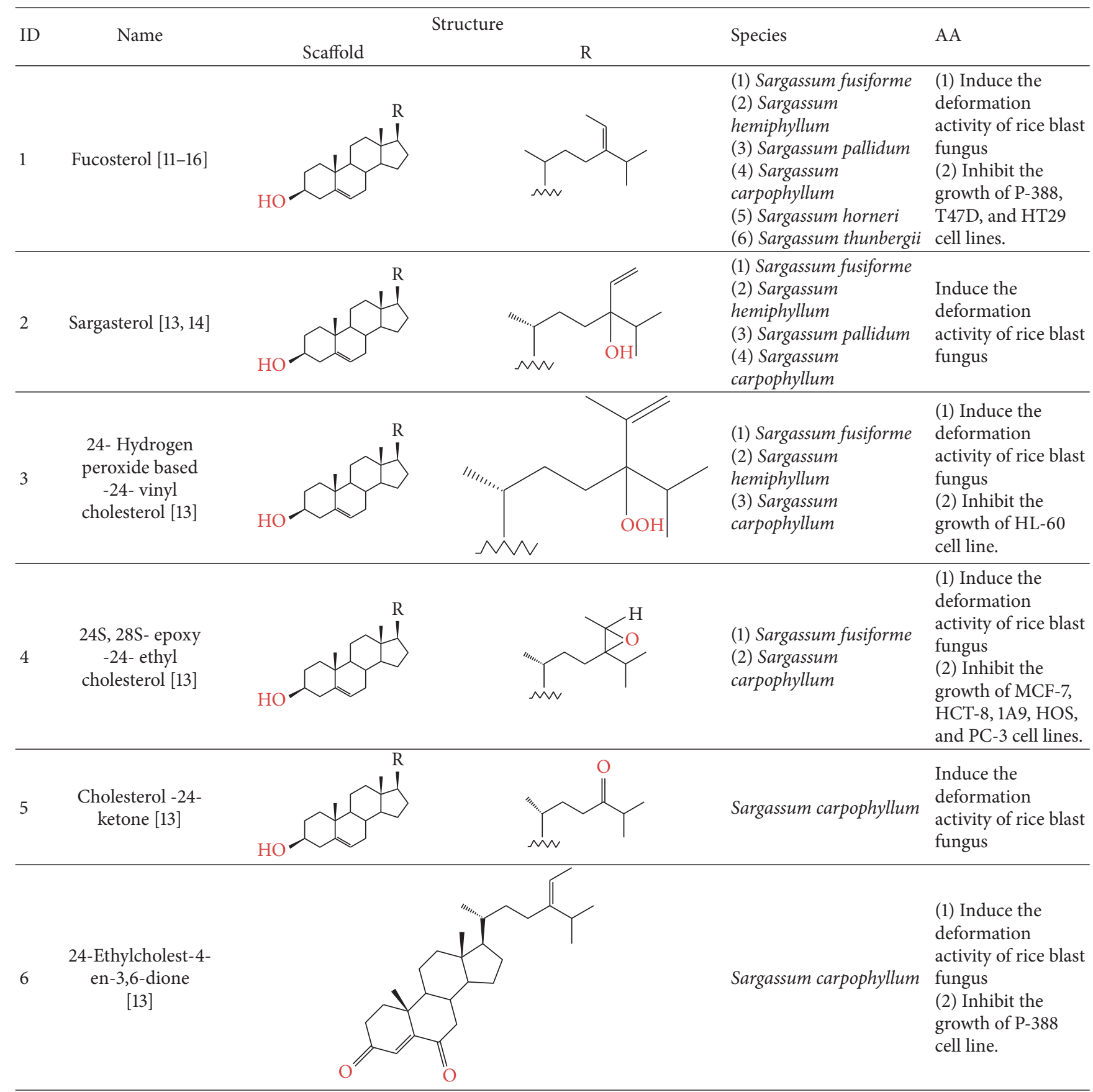

with cold nature such as Chlorophyta, Florideophyceae, and Phaeophyceae contain antitumor properties [51].

\section{Conclusions}

Our analysis demonstrated that potential AA derived from Metazoa or Viridiplantae species with cold nature tended to have close taxonomic relationship than distantly distributed in phylogenetic tree. The clustered patterns with mined association rules presented in this work provide information pertaining to the groups of species with anticancer properties. Moreover, we have shown that phylogenetic tree analysis can be utilized to shortlist plant or animal species that possess potential AA. Future bioprospecting studies on TCMMs are thus warranted with aims of producing novel anticancer drugs.

\section{Competing Interests}

The authors declare no competing financial interest. 


\section{Authors' Contributions}

Xianjun Fu conceived the main theme on which the work is performed and ensured that the scientific aspect of the study is rationally valid and dedicated a substantial contribution to the conception and design of the project and analysis and interpretation of the data and wrote this manuscript. Xuxia Song and Jiaoyang Li contributed in data preparation. Kah Keng Wong, Xuebo Li, Fengcong Zhang, and Changyun Wang helped in the analysis and interpretation of data. Zhenguo Wang ensured that the scientific aspect of the study was rationally valid. All authors have read and approved the final manuscript.

\section{Acknowledgments}

This work is supported by the National Natural Science Foundation of China (Grant no. 81473369).

\section{References}

[1] X.-J. Fu, Z.-G. Wang, Y. Qu, P. Wang, Y. Zhou, and H.-Y. $\mathrm{Yu}$, "Study on the networks of 'nature-family-component' of Chinese medicinal herbs based on association rules mining," Chinese Journal of Integrative Medicine, vol. 19, no. 9, pp. 663667, 2013.

[2] F. Liang, L. Li, M. Wang et al., "Molecular network and chemical fragment-based characteristics of medicinal herbs with cold and hot properties from Chinese medicine," Journal of Ethnopharmacology, vol. 148, no. 3, pp. 770-779, 2013.

[3] C. Zhou, J. Wang, X. Zhang et al., "Investigation of the differences between the 'COLD' and 'HOT' nature of Coptis chinensis Franch and its processed materials based on animal's temperature tropism," Science in China Series C: Life Sciences, vol. 52, no. 11, pp. 1073-1080, 2009.

[4] P. P. Li, "Toward an integrative framework of indigenous research: the geocentric implications of Yin-Yang Balance," Asia Pacific Journal of Management, vol. 29, no. 4, pp. 849-872, 2012.

[5] T. Ma, C. Tan, H. Zhang, M. Wang, W. Ding, and S. Li, "Bridging the gap between traditional Chinese medicine and systems biology: the connection of cold syndrome and NEI network," Molecular BioSystems, vol. 6, no. 4, pp. 613-619, 2010.

[6] F. Mohd Fauzi, A. Koutsoukas, R. Lowe et al., "Chemogenomics approaches to rationalizing the mode-of-action of traditional chinese and ayurvedic medicines," Journal of Chemical Information and Modeling, vol. 53, no. 3, pp. 661-673, 2013.

[7] L.-P. Huang, M. F. Zhu, R. Y. Yu, J. Q. Du, and H. N. Liu, "Study on discrimination mode of cold and hot properties of traditional chinese medicines based on biological effects," Zhongguo Zhongyao Zazhi, vol. 39, no. 17, pp. 3353-3358, 2014.

[8] H. Zhao, Y. Zhao, J. Wang et al., "Expression of the difference between the Cold (Han) and Hot (Re) natures of traditional Chinese medicines (Strobal and Rhubarb) based on the cold/hot plate differentiating assay," Science in China, Series C: Life Sciences, vol. 52, no. 12, pp. 1192-1197, 2009.

[9] X. Fu, H. Guan, W. Qiangming et al., "Elementary exploration of the orion and development of marine Chlnelle materla medica," Chinese Journal of Medical History, vol. 39, no. 3, pp. 168-172, 2009.
[10] X. Fu, M. Zhang, C. Shao et al., "Chinese marine materia medica resources: status and potential," Marine Drugs, vol. 14, no. 3, p. 46, 2016.

[11] M. Terasaki, A. Hirose, B. Narayan et al., "Evaluation of recoverable functional lipid components of several brown seaweeds (phaeophyta) from japan with special reference to fucoxanthin and fucosterol contents," Journal of Phycology, vol. 45, no. 4, pp. 974-980, 2009.

[12] S.-E. N. Ayyad, S. T. Ezmirly, S. A. Basaif, W. M. Alarif, A. F. Badria, and F. A. Badria, "Antioxidant, cytotoxic, antitumor, and protective DNA damage metabolites from the red sea brown alga Sargassum sp," Pharmacognosy Research, vol. 3, no. 3, pp. 160-165, 2011.

[13] H.-F. Tang, Y.-H. Yi, X.-S. Yao, Q.-Z. Xu, S.-Y. Zhang, and H.-W. Lin, "Bioactive steroids from the brown alga Sargassum carpophyllum," Journal of Asian Natural Products Research, vol. 4, no. 2, pp. 95-101, 2002.

[14] Y. M. Ham, K. N. Kim, W. J. Lee, N. H. Lee, and C. G. Hyun, "Chemical constituents from Sargassum micracanthum and antioxidant activity," International Journal of Pharmacology, vol. 6, no. 2, pp. 147-151, 2010.

[15] M. Khanavi, R. Gheidarloo, N. Sadati et al., "Cytotoxicity of fucosterol containing fraction of marine algae against breast and colon carcinoma cell line," Pharmacognosy Magazine, vol. 8, no. 29, pp. 60-64, 2012.

[16] M. Kobayashi, A. Hasegawa, and H. Mitsuhashi, "Marine sterols. XV. Isolation of 24-vinyloxycholesta-5,23-dien-3 $\beta$-ol from the brown alga Sargassum thumbergii," Chemical and Pharmaceutical Bulletin, vol. 33, no. 9, pp. 4012-4013, 1985.

[17] H. Yang, J. Li, Y. Zheng et al., "Drug activity screening based on microsomes-hydrogel system in predicting metabolism induced antitumor effect of oroxylin A," Scientific Reports, vol. 6, article no. 21604, 2016.

[18] M. Sugumaran and W. E. Robinson, "Bioactive dehydrotyrosyl and dehydrodopyl compounds of marine origin," Marine Drugs, vol. 8, no. 12, pp. 2906-2935, 2010.

[19] X. Fu, Z. Wang, C. Wang et al., "The distribution and association relationships of marine Chinese medicine with different nature in the phylogenetic tree of marine organisms," Modernization of Traditional Chinese Medicine and Materia Medica. World Science and Technology, vol. 17, no. 11, pp. 2189-2196, 2015 (Chinese).

[20] X. Fu, C. Pan, S. Lin et al., "Study on nature distribution of marine Chinese medicine based on literature mining," China Journal of Traditional Chinese Medicine and Pharmacy, vol. 31, no. 1, pp. 96-100, 2016.

[21] P. A. Harnedy and R. J. FitzGerald, "Bioactive peptides from marine processing waste and shellfish: a review," Journal of Functional Foods, vol. 4, no. 1, pp. 6-24, 2012.

[22] S. G. Guan and H. S. Wang, Chinese Marine Materia Medica, Shanghai Scientific and Technical Publishers, Shanghai, China, 2009.

[23] S. Singh, A. E. Carpenter, and A. Genovesio, "Increasing the content of high-content screening: an overview," Journal of Biomolecular Screening, vol. 19, no. 5, pp. 640-650, 2014.

[24] F. Zhu, C. Qin, L. Tao et al., "Clustered patterns of species origins of nature-derived drugs and clues for future bioprospecting," Proceedings of the National Academy of Sciences of the United States of America, vol. 108, no. 31, pp. 12943-12948, 2011.

[25] A. Mcbride and S. K. Butler, "Eribulin mesylate: a novel halichondrin $\mathrm{B}$ analogue for the treatment of metastatic breast 
cancer," American Journal of Health-System Pharmacy, vol. 69, no. 9, pp. 745-755, 2012.

[26] F. Zhu, X. H. Ma, C. Qin et al., "Drug discovery prospect from untapped species: indications from approved natural product drugs," PLoS ONE, vol. 7, no. 7, Article ID e39782, 2012.

[27] E. Pean, S. Klaar, E. G. Berglund et al., "The European Medicines Agency review of eribulin for the treatment of patients with locally advanced or metastatic breast cancer: summary of the scientific assessment of the committee for medicinal products for human use," Clinical Cancer Research, vol. 18, no. 17, pp. 4491-4497, 2012.

[28] D. L. Wheeler, T. Barrett, D. A. Benson et al., "Database resources of the national center for biotechnology information," Nucleic Acids Research, vol. 35, supplement 1, pp. D5-D12, 2007.

[29] F. D. Ciccarelli, T. Doerks, C. Von Mering, C. J. Creevey, B. Snel, and P. Bork, "Toward automatic reconstruction of a highly resolved tree of life," Science, vol. 311, no. 5765, pp. 1283-1287, 2006.

[30] H. Zhang, S. Gao, M. J. Lercher, S. Hu, and W.-H. Chen, "EvolView, an online tool for visualizing, annotating and managing phylogenetic trees," Nucleic Acids Research, vol. 40, no. 1, pp. W569-W572, 2012.

[31] I. Letunic and P. Bork, "Interactive Tree Of Life (iTOL): an online tool for phylogenetic tree display and annotation," Bioinformatics, vol. 23, no. 1, pp. 127-128, 2007.

[32] M. Hahsler, B. Grun, and K. Hornik, "The arules package: mining association rules and frequent itemsets, version 0.6-6," 2008.

[33] J. Fürnkranz, Association Rule, Encyclopedia of Systems Biology, Springer, New York, NY, USA, 2013.

[34] E. Dasseni, V. S. Verykios, A. K. Elmagarmid, and E. Bertino, "Hiding association rules by using confidence and support," in Information Hiding, vol. 2137 of Lecture Notes in Computer Science, pp. 369-383, Springer, Berlin, Germany, 2001.

[35] F. Lazcano-Pérez, S. A. Román-González, N. Sánchez-Puig, and R. Arreguín-Espinosa, "Bioactive peptides from marine organisms: a short overview," Protein and Peptide Letters, vol. 19, no. 7, pp. 700-707, 2012.

[36] G. Faircloth and M. del Carmen Cuevas Marchante, "Kahalalide F and ES285: potent anticancer agents from marine molluscs," in Molluscs: From Chemo-Ecological Study to Biotechnological Application, vol. 43 of Progress in Molecular and Subcellular Biology, pp. 363-379, Springer, Berlin, Germany, 2006.

[37] B. Pejin, M. Mojovic, and A. G. Savic, "Novel and highly potent antitumour natural products from cnidarians of marine origin," Natural Product Research, vol. 28, no. 24, pp. 2237-2244, 2014.

[38] N. G. M. Gomes, F. Lefranc, A. Kijjoa, and R. Kiss, "Can some marine-derived fungal metabolites become actual anticancer agents?" Marine Drugs, vol. 13, no. 6, pp. 3950-3991, 2015.

[39] L. A. Salvador-Reyes, N. Engene, V. J. Paul, and H. Luesch, "Targeted natural products discovery from marine cyanobacteria using combined phylogenetic and mass spectrometric evaluation," Journal of Natural Products, vol. 78, no. 3, pp. 486492, 2015.

[40] F. Leliaert, A. Tronholm, C. Lemieux et al., "Chloroplast phylogenomic analyses reveal the deepest-branching lineage of the Chlorophyta, Palmophyllophyceae class. nov.", Scientific Reports, vol. 6, Article ID 25367, 2016.

[41] X. Kai and M. Mingsan, "The characteristics and the modern research of potency cold," Chinese Journal of Chinese Medicine, vol. 28, no. 182, pp. 1006-1008, 2013.
[42] T. L. Simmons, E. Andrianasolo, K. McPhail, P. Flatt, and W. H. Gerwick, "Marine natural products as anticancer drugs," Molecular Cancer Therapeutics, vol. 4, no. 2, pp. 333-342, 2005.

[43] E. A. Gontang, S. P. Gaudêncio, W. Fenical, and P. R. Jensen, "Sequence-based analysis of secondary-metabolite biosynthesis in marine actinobacteria," Applied and Environmental Microbiology, vol. 76, no. 8, pp. 2487-2499, 2010.

[44] M. Wink and G. I. A. Mohamed, "Evolution of chemical defense traits in the Leguminosae: mapping of distribution patterns of secondary metabolites on a molecular phylogeny inferred from nucleotide sequences of the rbcL gene," Biochemical Systematics and Ecology, vol. 31, no. 8, pp. 897-917, 2003.

[45] C. W. Levy, A. Roujeinikova, S. Sedelnikova et al., "Molecular basis of triclosan activity," Nature, vol. 398, no. 6726, pp. 383384, 1999.

[46] D.-X. Kong, Y.-Y. Jiang, and H.-Y. Zhang, "Marine natural products as sources of novel scaffolds: achievement and concern," Drug Discovery Today, vol. 15, no. 21-22, pp. 884-886, 2010.

[47] V. J. Paul and W. Fenical, "Natural products chemistry and chemical defense in tropical marine algae of the phylum Chlorophyta," in Bioorganic Marine Chemistry, vol. 1 of Bioorganic Marine Chemistry, pp. 1-29, Springer, Berlin, Germany, 1987.

[48] Y. Pengzhan, Z. Quanbin, L. Ning, X. Zuhong, W. Yanmei, and L. Zhi'en, "Polysaccharides from Ulva pertusa (Chlorophyta) and preliminary studies on their antihyperlipidemia activity," Journal of Applied Phycology, vol. 15, no. 1, pp. 21-27, 2003.

[49] H. Noda, H. Amano, K. Arashima, and K. Nisizawa, "Antitumor activity of marine algae," Hydrobiologia, vol. 204-205, no. 1, pp. 577-584, 1990.

[50] F. Xianjun, W. Peng, and W. Zhenguo, "Hypothesis on building of chemical constituent element system of cold-heat nature based on study of 'nature-structure relationship' of traditional Chinese medicine," Modernization of Traditional Chinese Medicine and Materia Medica-World Science and Technology, vol. 13, no. 5, pp. 919-924, 2011.

[51] C. Xiao, D. Li, and H. Wang, "Medicinal composition of main economic algae in our China," Northwest Pharmaceutical Journal, vol. 23, no. 6, pp. 408-410, 2008. 


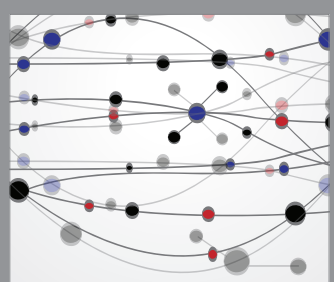

The Scientific World Journal
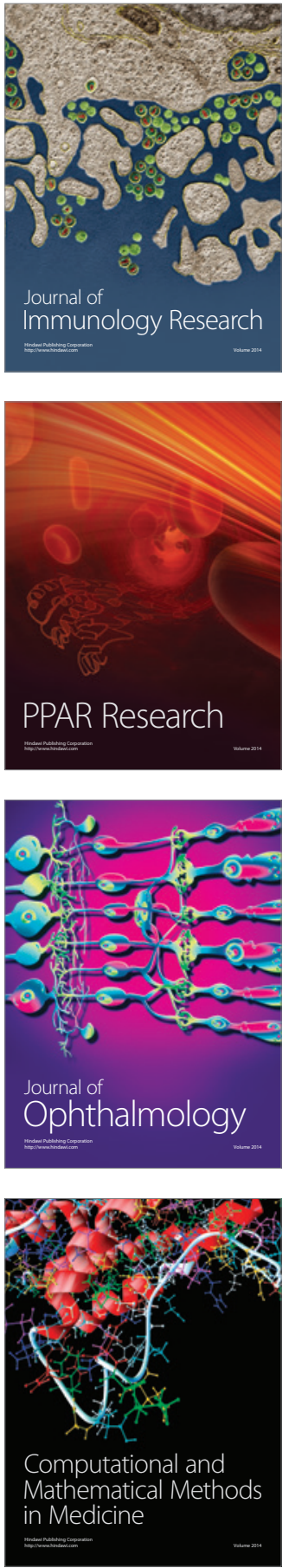

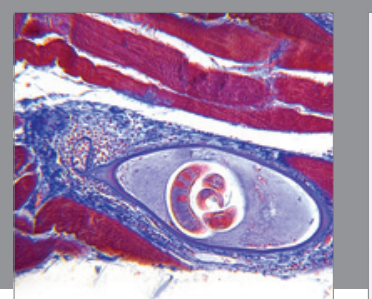

Gastroenterology Research and Practice
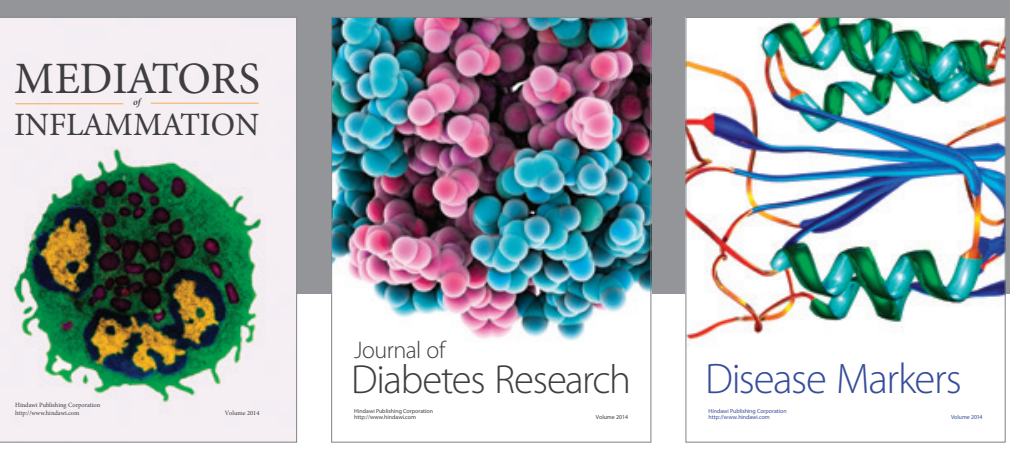

Disease Markers

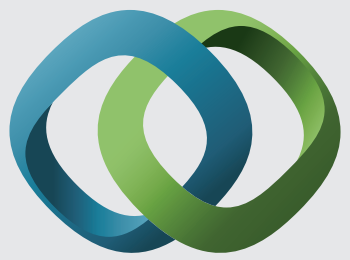

\section{Hindawi}

Submit your manuscripts at

https://www.hindawi.com
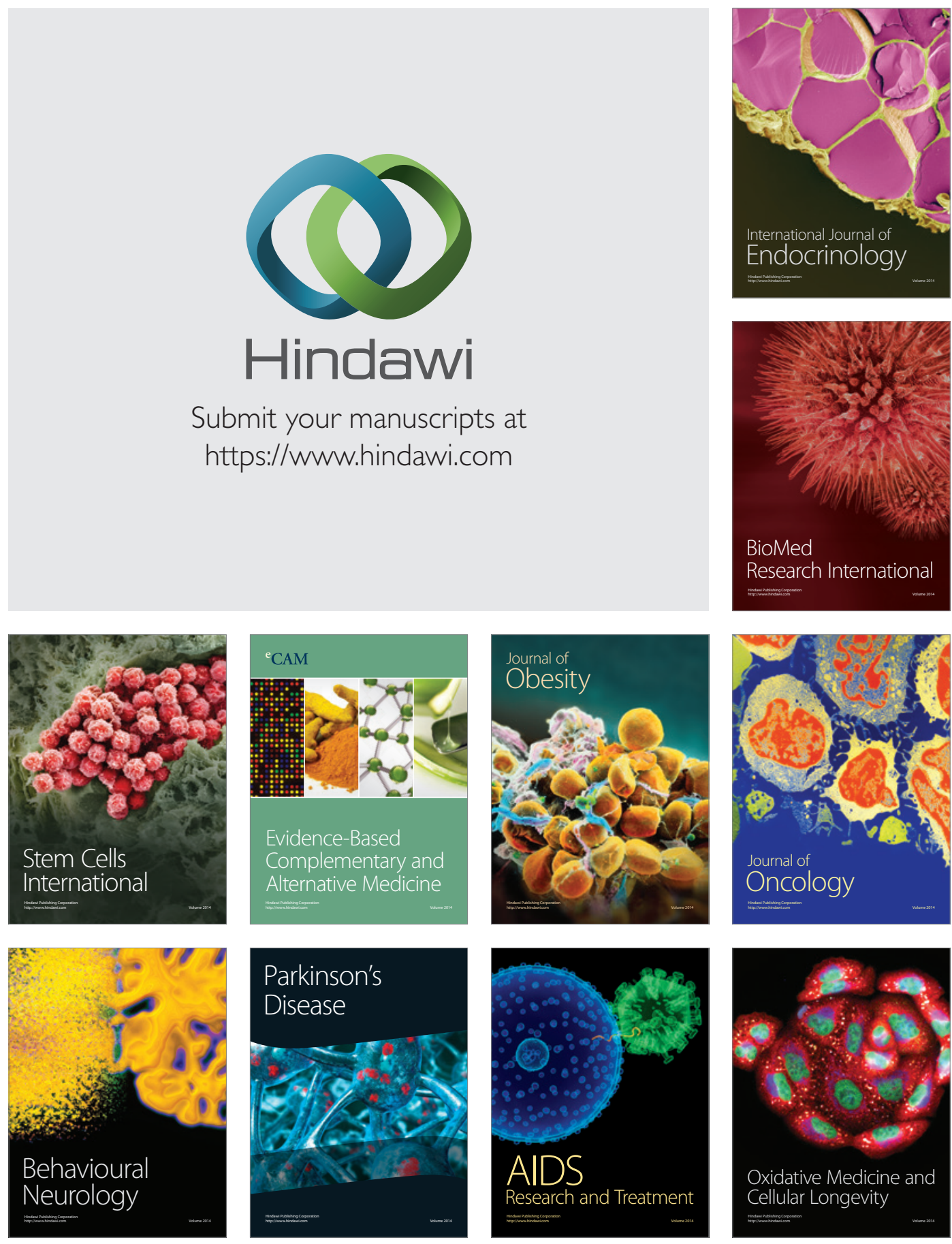